\title{
Discovery and the Culture of Astronomy
}

\author{
Robert Williams ${ }^{1}$ \\ Space Telescope Science Institute \\ Baltimore, MD USA \\ wmsestsci. edu
}

\begin{abstract}
The process by which discoveries are made and accepted is central to progress in astronomy. The manner in which papers are refereed, telescope time is awarded, and research funds are allocated evolves (at best) slowly with time, lacking a systematic procedure by which it is evaluated and modified. It would benefit from greater diversity, e.g., witness the great success of astro-ph. The culture by which astronomy is done should be subjected from time to time to the same scrutiny as research proposals and journal publications. Decadal surveys, in those countries that undertake them, should be a good mechanism for conducting such process assessment and recommending change.
\end{abstract}

Accelerating the Rate of Astronomical Discovery - sps 5

Rio de Janeiro, Brazil

August 11-14 2009

1 Speaker 
I was asked to start off this session on discovery by making general remarks that might provide a perspective for subsequent discussion. Let me begin by stating that the act of astronomical discovery per se is only a part of what is relevant to this session. In fact, it may be a small part. Agreeing on what constitutes discovery and assessing the process by which discovery occurs and is accepted are an integral part of accelerating discovery. One can point to 'discoveries' such as the Saha equation that were not initially accepted for publication and which were therefore delayed or denied. Discoveries have little significance as long as they remain only with the discoverer. The recent success of astro-ph with its largely unfiltered acceptance of manuscripts has proven quite effective in the dissemination of ideas, contrasting as it does with the refereed journals. This raises interesting questions. Is astro-ph successful because of the journals? Are there checks and balances that will prevent it from evolving into electronic babble?, i.e., a diversion akin to television talk shows? Clearly, the entire process by which research is done, i.e., the culture of science, is central to discovery. In addition to fostering creative spark, accelerating discovery involves optimizing the research process.

Astronomical discoveries are made through observations and their interpretation, and through theoretical results that follow from fundamental principles and which are verified by observations. These require knowledgeable individuals who generally have need for funding. Consider some of the components involved in this process. First, education. To take just one example, graduate curricula have in the past often concentrated on basic physics/astrophysics and observational techniques to the detriment of instrumentation development. This situation has been recognized and addressed in recent years and there are now more graduate programs offering a specialization in instrumentation. This is an important step in achieving a balance that should be more conducive to discoveries.

Research infrastructure is one of the most important catalysts for discovery. New phenomena are inevitably discovered when novel equipment and techniques are applied to observations. However, creating and maintaining a forefront infrastructure is costly and it creates the debate that large, innovative projects divert funds from more traditional infrastructure development that supports the work of the broader community. This competition for resources within the community is inevitable and healthy, and is usually dealt with by the peer review process. On a larger scale the competition for funds has motivated some countries to undertake decade reviews in order to set priorities.

Are there criteria that we can agree upon that can be invoked when making major funding decisions among competing groups? Later today two of the sessions of this Spec. Session will be devoted to some questions that some of us have wrestled with (1) concentration of large facilities at national centers vs. smaller facilities from university departments, and (2) what is the most effective way to assign time on major telescopes? Simply on grounds of lack of diversity one could question whether the current reliance of telescope time allocation on peer review committees is optimal because so many different observatories have adopted the same system. In a world in which the e-folding time for change of everything is of order ten years, it is healthy to be skeptical of any 
process that has remained unchanged for more than a generation. Most peer review processes have remained basically the same for the past half century. It is important to have a mechanism for assessing and optimizing process by making modifications. I remain puzzled (read that: frustrated) by the reluctance of communities to give a greater fraction of telescope time to the director of a facility to assign, especially when that director reports to a council and is him/herself reviewed regularly. High risk, high reward projects require hard decisions. Hard decisions are more readily made by individuals, not committees. At least $20-25 \%$ of time should be awarded by the director of a facility. Such a policy would definitely accelerate discovery.

Funding and publication are critical elements in discovery, and both of them involve peer review. The peer review system has evolved over the years to its current state. Is it the most effective system that it can be in supporting discovery? It would be useful to develop criteria by which the effectiveness of the peer review system can be assessed.

One of the dogmas of science is that novel ideas tend to emanate from single individuals. Committees have a tendency to minimize risk. Individuals are less constrained. It is noteworthy that the peer review system for publication has evolved differently than that for funding and telescope time assignment. The journal refereeing system relies primarily on a single individual, the referee, who the editor believes is knowledgeable about the subject of the manuscript. By contrast, funding decisions are generally recommended by committees of many individuals representing a broad range of expertise. There are reasons for this difference, e.g., the amount of funds available is usually limited whereas there is no hard limit to the number of journal pages that can be published. But, it does call attention to the fact that different circumstances may call for different processes, and these processes are a sufficiently important part of doing science that they merit analysis and scrutiny. Better to exercise some control over them than to simply let them passively evolve.

When possible, metrics should be developed that will aid in the assessment of process. Consensus may be difficult to achieve on their validity so they may not be decisive in establishing the best courses of action in a situation where subjective judgment tends to rule. Therefore, experimentation with different processes may be more effective and should be encouraged, as this is likely to be the best way of arriving at good processes. Such experimentation may, in fact, be an important reason why the present peer review system and culture in astronomy is so uniform world-wide. The community should be flexible in accepting different procedures for similar activities in different organizations.

Many of us are familiar with an environment where every aspect of a project is subjected to review in a process called program management. All components of a project are subjected to cost vs. benefit analysis. I would not advocate this on a continuing basis for process or culture, however I do think it makes sense to subject important aspects of the culture of doing science to periodic analysis. It is important that we ask questions about every facet of how we advance discovery, and then find ways to make corrections to those processes. Let's subject to occasional scrutiny such issues as: (a) to what extent should national centers give telescope time to private groups who provide new instrumentation 
at their expense? (b) should the terms of postdocs be limited to 3 years? (c) do tenure decisions require 10-15 external letters of reference? (d) would acceptance of journal papers, i.e., evaluation of the referee's report(s) and author's response(s) be better made by an independent referee rather than the editor? (e) Under what conditions is the national community served best by management organizations such as AURA, AUI, USRA, etc. that have large numbers of members, including international members, as opposed to smaller, more cohesive university management organizations? We can disagree on the answers to these questions, but I find it hard to argue against creating within our astronomy culture forums where these questions are raised and aired out.

Cultures are very hard to change, which is not a bad thing since their evolution is normally driven by the community good. The difficulty occurs when the environment changes faster than the culture. There is good reason to have a mechanism in place by which change in process can be an integral part of science culture. At some level serious attention should be paid to creating a process by which questions are asked of traditional procedures, and assessments and recommendations made. As one concrete suggestion I would advocate that those countries that undertake decade surveys should address the question of astronomy culture and process. The broad community participation in the decade reviews and the cachet that they have with all segments of the community---from scientists and administrators, funding agencies, and government officials---makes them an ideal vehicle for assessing astronomy culture and recommending change where it might accelerate the progress of science. 